\title{
Three-dimensional simplified electrodynamic model of the human chest
}

\author{
Alexander Dem'yanenko ${ }^{1, *}$, Yaroslav Nevstruev ${ }^{1}$, and Olga Semernik ${ }^{2}$ \\ ${ }^{1}$ Southern Federal University, Department of Antennas and Radio Transmitting Devices, 347922 \\ Taganrog, Russia \\ ${ }^{2}$ Rostov State Medical University, Department of Childhood Diseases No. 2, 344022 Rostov-on-Don, \\ Russia
}

\begin{abstract}
The results of the development of an accessible threedimensional electrodynamic model of the human chest in a healthy state and in the presence of various pathologies caused by bronchopulmonary diseases are presented. Simulation and experimental study of the propagation of electromagnetic waves in the human chest has been carried out with the aim of developing high-tech harmless non-invasive methods, devices and automated systems for diagnosing diseases of the bronchopulmonary system based on modern microwave technologies.
\end{abstract}

\section{Introduction}

One of the leading positions in the list of the most common diseases is respiratory diseases, the most serious of which are bronchial asthma and pneumonia [1,2]. Despite modern scientific achievements to date have not been developed methods and devices to carry out a harmless, non-invasive diagnosis of changes in the bronchopulmonary system, caused by the disease.

Currently, the most common methods for diagnosing bronchopulmonary diseases in clinical practice are radiological (fluorography, fluoroscopy, radiography, computed tomography). The main disadvantage of these methods is the low sensitivity to the presence of sputum in the lungs, which is detected when it is more than $30 \%$ increase from the normal state. Also disadvantages are exposure to ionizing radiation, the high cost of examination.

Every year in medicine, radio and microwave radiation is used more actively [3]. The main areas of application of microwave radiation are measuring the fluid content in human tissues, detecting breast cancer, studying the movement of the arterial wall, observing cardiac and nervous activity, as well as non-invasive therapy by heating tissues when exposed to microwave radiation [4-6]. However, there is currently no commercially available device that would allow for the non-invasive diagnosis of diseases of the bronchopulmonary system based on microwave methods.

To develop modern high-tech methods for diagnosing diseases of the bronchopulmonary system, as well as automated systems for diagnosing diseases and

\footnotetext{
* Corresponding author: demalex@inbox.ru
} 
monitoring the condition of patients, electrodynamic models are needed, on the basis of which the methods themselves will be developed, as well as testing and testing the effectiveness of diagnostic devices that implement these methods. In addition, fundamental research is needed on the propagation of radio waves and microwave signals of various types in the human chest in a healthy condition and in the presence of one or another pathology.

This paper presents the results of a scientific study aimed at developing affordable electrodynamic models of the human chest in a healthy state and in the presence of various pathologies caused by bronchopulmonary diseases, as well as modeling and experimental study of the propagation of electromagnetic waves and microwave signals of various types, including sinusoidal pulsed and chaotic in the human chest in order to develop high-tech harmless non-invasive methods, mouth systems and automated systems for diagnosing diseases of the bronchopulmonary system, based on modern microwave technologies.

\section{Research methods}

To develop three-dimensional electrodynamic models, we used methods for synthesizing electrodynamic models from empirical data obtained by statistical analysis of the results of X-ray studies, MRI and CT studies of the human chest in a healthy state and in the presence of pathological changes caused by bronchopulmonary diseases.

A comprehensive examination of patients with bronchopulmonary pathology (acute simple bronchitis, obstructive bronchitis, bronchiolitis, various forms of communityacquired pneumonia and bronchial asthma) using standard techniques (studying clinical and medical history data, studying laboratory parameters, X-ray examination, peak flow measurement, spirography, bodypletismography) allows you to get data for the development of three-dimensional electrodynamic models of the human chest in a healthy state and in the presence of pathologies any severity.

Based on the data of the examination and analysis, the overall parameters of the tissues and organs that make up the chest are determined. Thus, the overall parameters of threedimensional electrodynamic models are as close as possible to the real parameters of the human chest (Fig. 1).

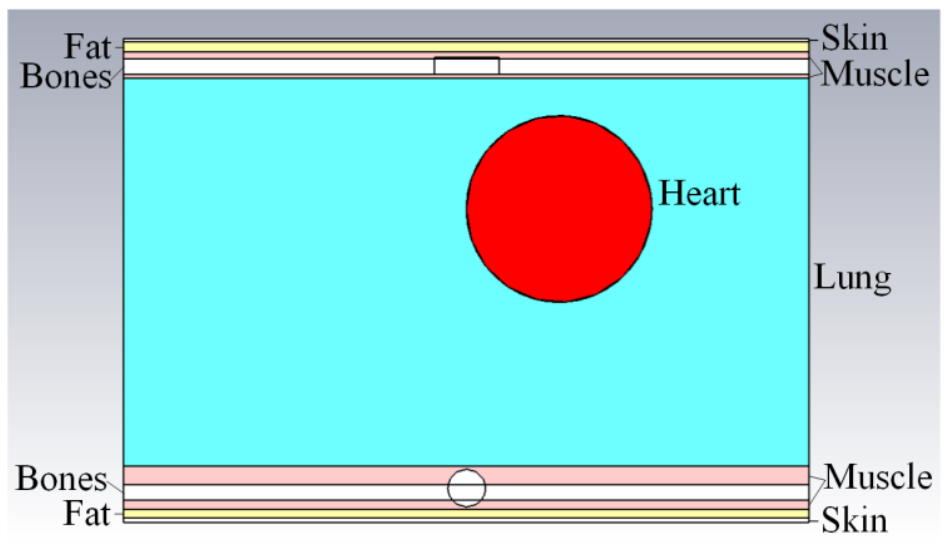

Fig. 1. Structure of the chest area model in CST STUDIO.

The electrophysical parameters of tissues and organs in the electrodynamic model of the human chest correspond to the real values of the indicated parameters of the tissues of the human body, which ensures that the simulation results are close to the real conditions observed during the experimental study. 
To achieve this goal, methods of electrodynamic modeling of electromagnetic fields using modern computer-aided design (CAD) systems, including CST STUDIO (Fig. 2), are used.

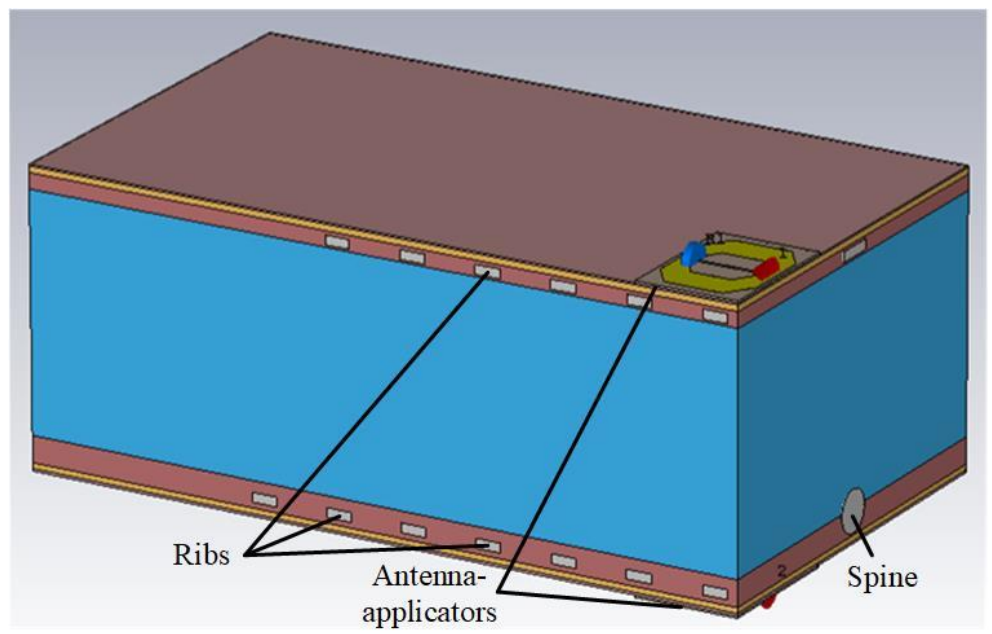

Fig. 2. Model of the chest area, with antenna applicators.

The constructed model does not aim to completely repeat the internal structure of the human chest, but is intended to identify the basic principles of the propagation of electromagnetic waves when taking into account internal heterogeneities.

The propagation of the electromagnetic field in the constructed model was studied using two applicator antennas [7,8], located opposite each other in direct contact with the chest model (Fig. 2), in order to minimize the reflection of electromagnetic radiation (EMR) from the surface of the chest. The design and overall dimensions of the used antenna applicators are optimized for the best radiation and reception of electromagnetic radiation in the frequency range from 900 to $1500 \mathrm{MHz}$ [9].

An analysis of the propagation of electromagnetic waves of the microwave range in the model and experimental phantom of the human chest will allow us to determine their effectiveness for non-invasive diagnosis of bronchopulmonary diseases and to develop appropriate diagnostic methods and devices.

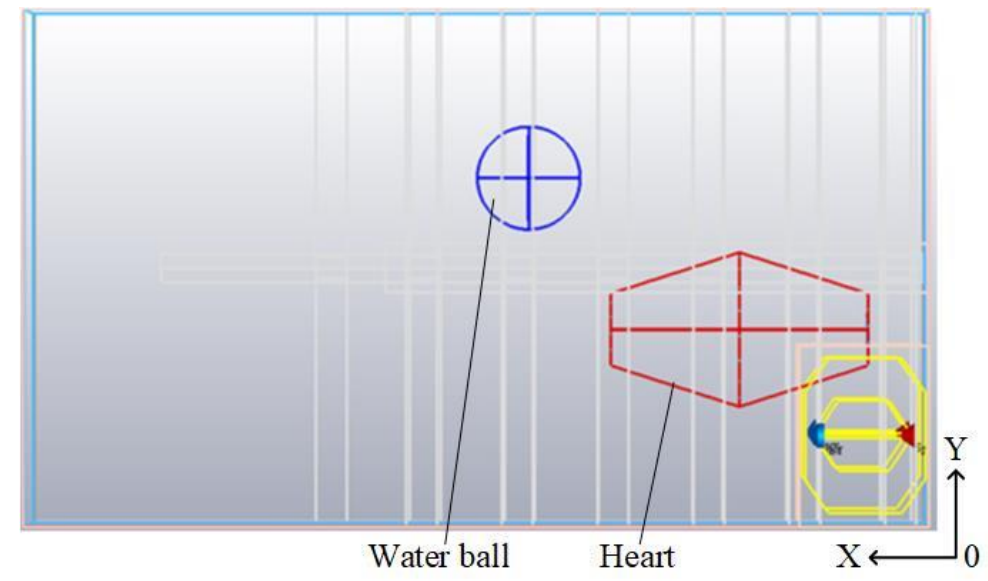

Fig. 3. Model of the chest area containing heterogeneity. 


\section{Results}

The propagation of electromagnetic waves was simulated in the frequency range from $900 \mathrm{MHz}$ to $1500 \mathrm{MHz}$. Thus, in the process of modeling and experimental research, the entire frequency range of the probing signals is blocked [10], within which it is possible to ensure accurate localization of changes in the human lung parenchyma caused by a particular disease, and harmless diagnosis of diseases of the bronchopulmonary system.

In this work, we measured the phase of the coefficient of transmission of the microwave signal through the chest by radio-frequency scanning, that is, the synchronous movement of the receiving and transmitting applicators on the surface of the model. Fig. 3 shows the studied model of the chest containing heterogeneity of a spherical shape with a volume of $33 \mathrm{ml}$, which corresponds to a diameter of heterogeneity of $22 \mathrm{~mm}$, simulating an excessive accumulation of sputum in the human chest.

Scanning was performed with a step of $10 \mathrm{~mm}$ along the $\mathrm{Y}$ axis, and with a step of $15 \mathrm{~mm}$ along the $\mathrm{X}$ axis.

In Fig. 4 shows the results of measuring the phase of the transmission coefficient obtained by radio-frequency scanning of the model of the chest area with a harmonic microwave signal with a frequency of $900 \mathrm{MHz}$ in the absence of heterogeneity (Fig. 4a) and in the presence of heterogeneity (Fig. 4b), simulating the presence of excessive phlegm in the phantom human chest. The black dots mark the places where the phase of the transmission coefficient of the microwave signal was measured during scanning. The simulation results, supplemented by the coordinates of the transmitting applicator, are processed in MATLAB. After the scan was completed, the results were approximated and visualized.
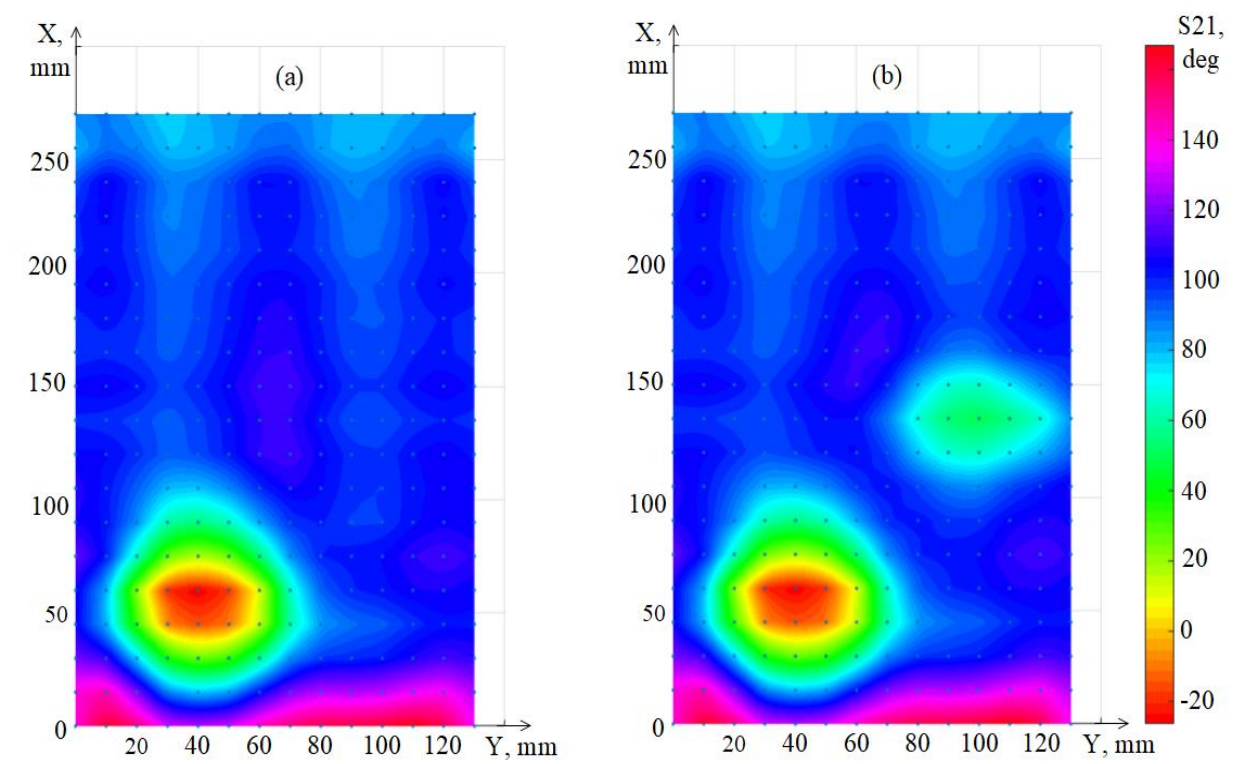

Fig. 4. Simulation results of the phase of the transmission coefficient estimation with a $900 \mathrm{MHz}$ signal without heterogeneity (a) and with heterogeneity (b).

From Fig. 4a shows that in the presence of only natural heterogeneities (heart, spine, etc.) in the model of the human chest, the phase of the transmission coefficient (S21) is predominantly uniform and approximately equal to 100 degrees, with the exception of the region of the location of the heart in which a decrease in the phase of the coefficient passing up to -20 degrees. Ribs and spine do not significantly affect the result. 
From Fig. $4 \mathrm{~b}$ it is seen that in the region of the location of the heterogeneity there is a change in the phase of the transmission coefficient up to 50 degrees, which allows one to determine the location of the heterogeneity and estimate its size and shape.

In Fig. 5 presents the results of measuring the phase of the transmission coefficient obtained by radio-frequency scanning of the model of the chest area with a harmonic microwave signal with a frequency of $1200 \mathrm{MHz}$ in the absence of heterogeneity (Fig. 5a) and in the presence of heterogeneity (Fig. 5b), simulating the presence of excessive phlegm in the phantom human chest.
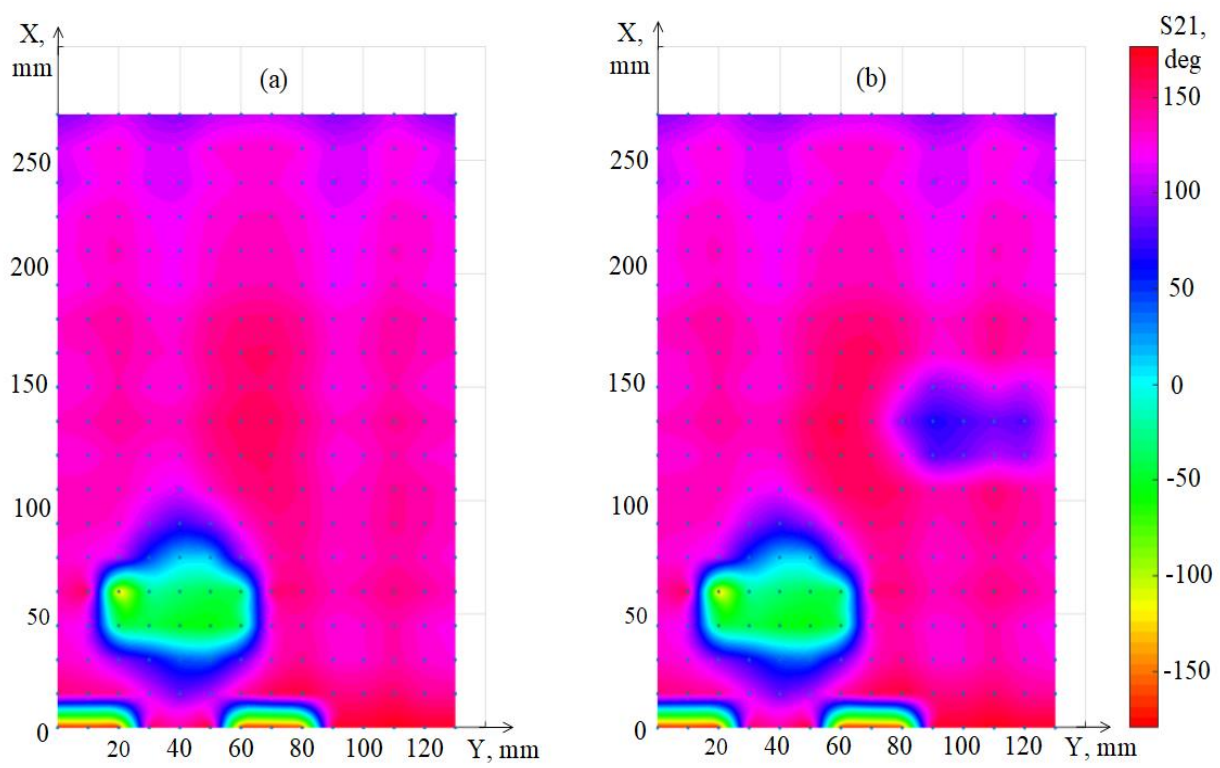

Fig. 5. Simulation results of the phase of the transmission coefficient estimation with a $1200 \mathrm{MHz}$ signal without heterogeneity (a) and with heterogeneity (b).

From Fig. 5a it is seen that in the presence of only natural inhomogeneities, the phase of the transmission coefficient (S21) is also uniform, but its value at a frequency of $1200 \mathrm{MHz}$ has become on average 110 degrees. The location of the heart is clearly visible, but it is not possible to correctly determine the shape from these results.

From Fig. 5b, it can be seen that in the area of the heterogeneity imitating sputum, the phase of the transmission coefficient has become equal to 50 degrees. Compared with the result obtained for a frequency of $900 \mathrm{MHz}$, the sputum position region has expanded, but the exact form of heterogeneity in this figure cannot be determined.

In Fig. 6 shows the results of measuring the phase of the transmission coefficient obtained by radio-frequency scanning of the model of the chest area with a harmonic microwave signal with a frequency of $1500 \mathrm{MHz}$ in the absence of heterogeneity (Fig. 6a) and in the presence of heterogeneity (Fig. 6b), simulating the presence of excessive phlegm in the phantom human chest.

From Fig. 6a it is seen that in the presence of only natural heterogeneities, the transmission coefficient has become less uniform than in the previous results. There were side areas with a phase of 130 degrees, which cannot be distinguished from that in which the heart and heterogeneity imitating sputum are located, as a result of which it is not possible to accurately determine their location in the chest.

A further increase in the frequency of the study leads to an even greater deterioration of the results, due to an increase in the absorption of the microwave signal in the human chest model. 

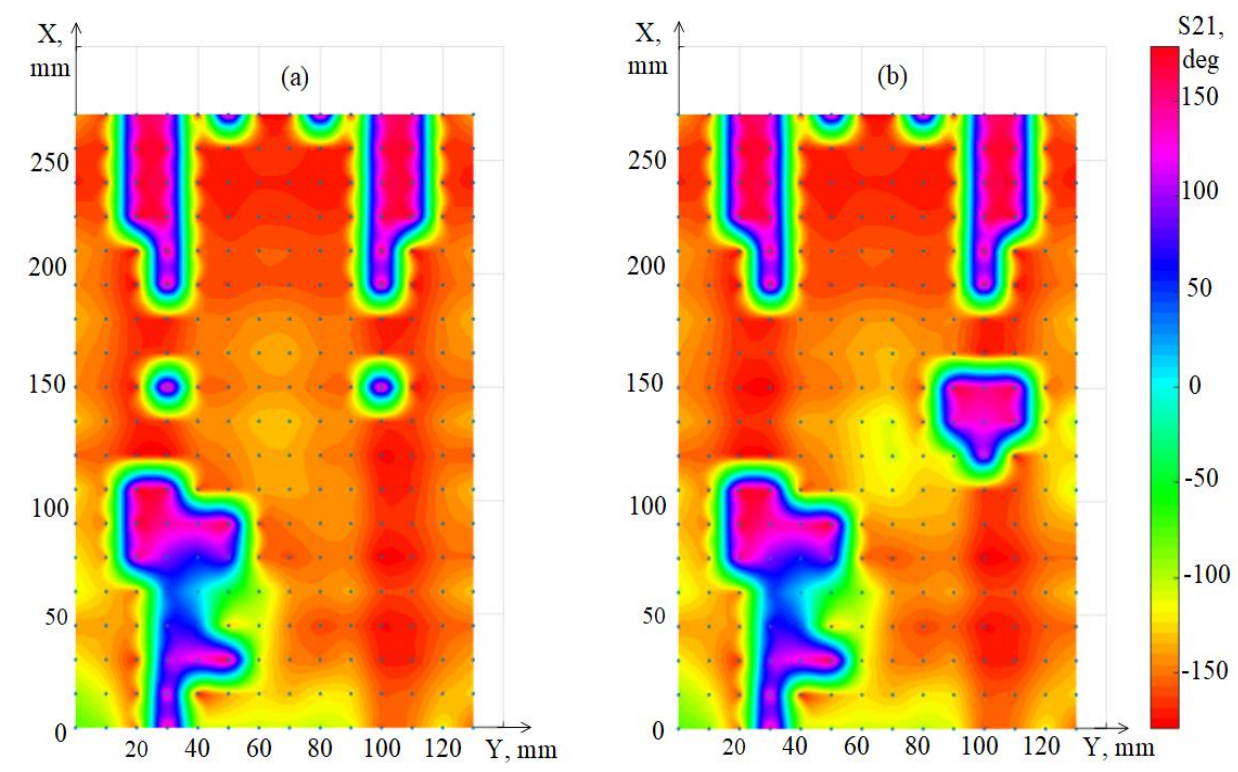

Fig. 6. Simulation results of the phase of the transmission coefficient estimation with a $900 \mathrm{MHz}$ signal without heterogeneity (a) and with heterogeneity (b).

\section{Comparison of results}

A comparison was made with the results of a previous study [11], in which the amplitude of the transmission coefficient was measured in a similar model of a section of the chest with a heterogeneity that mimics excessive accumulation of sputum.

In Fig. 7 shows the results of estimation the amplitude (a) and phase (b) during the simulation transmission coefficient obtained by radio-frequency scanning of the model of the chest area with a harmonic microwave signal with a frequency of $900 \mathrm{MHz}$ in the presence of heterogeneity, simulating excessive accumulation of sputum.

From Fig. 7 shows that the image obtained when measuring the phase of the transmission coefficient (a) at a frequency of $900 \mathrm{MHz}$ allows to more accurately determine the location and shape of the heterogeneity than by measuring the amplitude of the transmission coefficient (b).

In Fig. 8 shows the results of estimation the amplitude (a) and phase (b) during the simulation transmission coefficient obtained by radio-frequency scanning of a model of a section of the chest with a harmonic microwave signal with a frequency of $1200 \mathrm{MHz}$ in the presence of heterogeneity, simulating excessive accumulation of sputum.

From Fig. 8 shows that the obtained results of the transmission coefficient are comparable with each other, but the shape and position of the inhomogeneity can be most accurately determined from phase measurements (b).

In Fig. 9 shows the results of estimation the amplitude (a) and phase (b) during the simulation transmission coefficient obtained by radio-frequency scanning of a model of a section of the chest with a harmonic microwave signal with a frequency of $1500 \mathrm{MHz}$ in the presence of heterogeneity that simulates excessive accumulation of sputum.

From Fig. 9 shows that the results of estimation of the amplitudes of the transmission coefficient (a) are more uniform, as a result of which it is possible to determine the shape and location of the heterogeneity most accurately from them. 

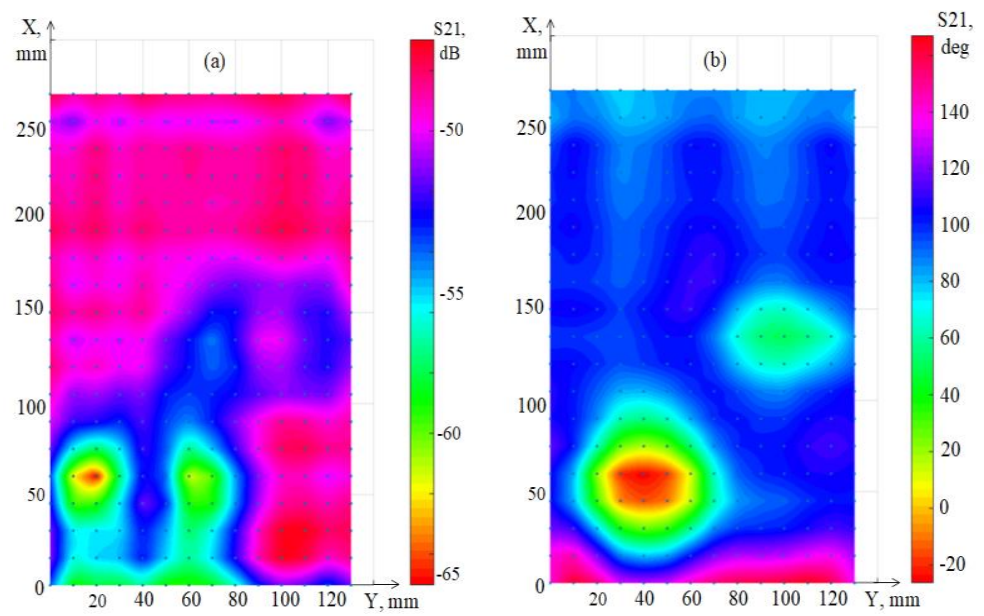

Fig. 7. Measurement results of the amplitude (a) and phase (b) of the transmission coefficient with a $900 \mathrm{MHz}$ signal.

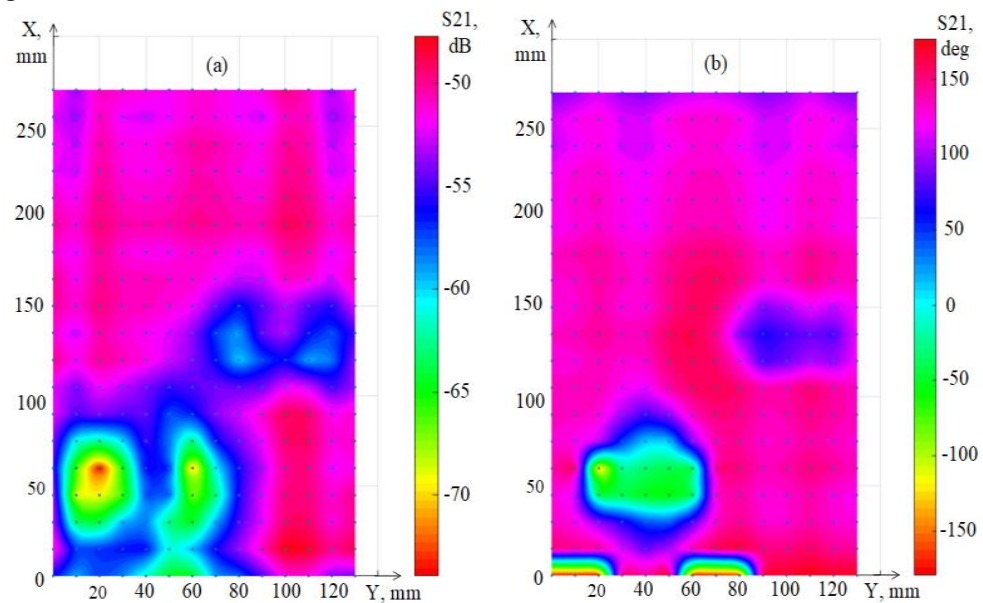

Fig. 8. Measurement results of the amplitude (a) and phase (b) of the transmission coefficient with a $1200 \mathrm{MHz}$ signal.
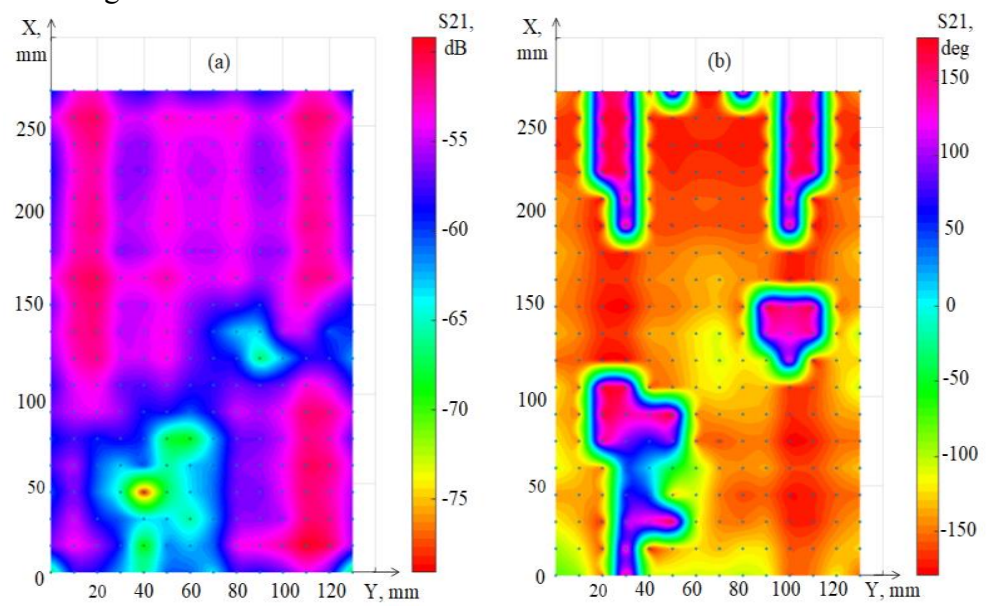

Fig. 9. Measurement results of the amplitude (a) and phase (b) of the transmission coefficient with a signal of $1500 \mathrm{MHz}$. 
Thus, based on the results of the comparison, it can be noted that the estimation of the phase of the transmission coefficient contributes to a more accurate determination of the shape and location of the heart and heterogeneity, simulating excessive accumulation of sputum up to a frequency of $1500 \mathrm{MHz}$. When estimating the amplitude of the transmission coefficient, the best result is observed at a frequency of $1500 \mathrm{MHz}$.

\section{Conclusion}

A three-dimensional electrodynamic model of the human chest is presented with sufficient detail for testing the designed components of diagnostic and monitoring devices based on the use of electromagnetic radiation. The level of detail is limited by the suitability for the further manufacture of the phantom of the human chest in a healthy condition and in the presence of pathologies using $3 \mathrm{D}$ printing technologies.

The obtained results of electromagnetic modeling indicate the possibility of using the proposed model of the human chest for testing methods and devices for diagnosing and monitoring the condition of a patient suffering from bronchopulmonary diseases, which are based on the use of electromagnetic radiation.

The study was carried out with the financial support of the Russian Foundation for Basic Research in the framework of the research project No.18-37-20045\18.

\section{References}

1. National Heart Lung and Blood Institute, "What is Asthma?," NIH [Online]. Available: http://www.nhlbi.nih.gov/health/health-topics/topics/asthma (2012)

2. Centers for Disease Control and Prevention, "Asthma," [Online]. Available: http:/www.cdc.gov/asthma/faqs.htm (2012)

3. S. Ahdi Rezaeieh, A. Zamani, K. S. Bialkowski, A. Mahmoud, A.M. Abbosh, Feasibility of Using Wideband Microwave System for Non-Invasive Detection and Monitoring of Pulmonary Oedema, Scientific Reports, v. 5(19), pp. 1-11 (2015)

4. V.S. Sokolova, A.B. Klimov, M.V. Gass, A.A. Svirin, The device for the study of blood supply and ventilation, Biomedical Technologies and Radio Electronics, № 3, pp. 3-7 (2003) (in Russian)

5. I.V. Semernik, A.V. Dem'yanenko, F.S. Topalov, Y.V. Nevstruev, O.E. Semernik, A.A. Lebedenko, An automated system for the early diagnosis of pathological changes in the bronchopulmonary system based on radio frequency scanning, Journal of Electronics, № 3 DOI: 10.30898/1684-1719.2019.3.6 [Online]. Available: http://jre.cplire.ru/jre/mar19/6/text.pdf (2019)

6. I.V. Semernik, A.V. Dem'yanenko, F.S. Topalov, O.E. Semernik, A.A. Lebedenko, Complex System for Monitoring the Patient's Condition and Diagnosis of Bronchial Asthma, Journal of Biomedical Physics and Engineering, pp. 1-8 DOI: 10.31661/ jbpe.v0i0.1022 (2019)

7. I.V. Semernik, A.V. Dem'yanenko, Y.V. Nevstruev, Development of a broadband microwave applicator for the diagnosis of bronchopulmonary diseases, 28th International Crimean Conference Microwave Technology and Telecommunication Technologies, pp. 1487-1493 (2018) (in Russian)

8. N. Celik, R. Gagarin, J. Baker, H. Youn, M.F. Iskander, A Noninvasive Microwave Sensor and Signal Processing Technique for Continuous Monitoring of Vital Signs, IEEE Antennas and Wireless Propagation Letters, v. 10, pp. 286-289 DOI: 10.1109/LAWP.2011.2132690 (2011) 
9. A.V. Dem'yanenko, I.V. Semernik, Y.V. Nevstruev, Designing of broadband microwave applicator for the bronchial asthma diagnosis device, Proceedings of 2018 IEEE Conference of Russian Young Researchers in Electrical and Electronic Engineering (EIConRus), pp. 1199-1202 DOI: 10.1109/EIConRus.2018.8317306 (2018)

10. I.V. Semernik, O.E. Semernik, A.V. Dem'yanenko, A.A. Lebedenko, Methods of noninvasive diagnosis of bronchial asthma in patients of all age groups based on microwave technology, Medical equipment, № 2, pp. 35-38 DOI: 10.1007/s10527017-9698-3 (2017)

11. Y.V. Nevstruev, Propagation of Microwave Electromagnetic Waves in the Human Thorax, 2019 Radiation and Scattering of Electromagnetic Waves (RSEMW), pp. 212215 DOI: 10.1109/RSEMW.2019.8792686 (2019) 